\title{
Relación existente entre la ratio 2D:4D y la composición corporal en adolescentes de Extremadura
}

\section{Relationship between 2D:4D ratio and body composition in adolescents in Extremadura}

\author{
Maria Mendoza Muñoz, Juan Manuel Franco García, David Gallego-Tena', Jorge \\ Carlos Vivas ${ }^{1}$. \\ ${ }^{1}$ Health, Economy, Motricity and Education (HEME) Research Group, Facultad de Ciencias del \\ Deporte, Cáceres, España.
}

\begin{abstract}
Resumen
Introducción: La ratio 2D:4D es el cociente entre la longitud del dedo índice y anular de la mano, asociada al balance entre andrógenos y estrógenos, durante el desarrollo embrionario. Una baja proporción 2D:4D, refleja niveles más altos de testosterona en el útero. Esta medida se ha relacionado con ciertos parámetros de la composición corporal, que podrían ser indicativos de la probabilidad de presentar riesgos para la salud. Objetivos: analizar si existe relación entre la ratio 2D:4D y la composición corporal y explorar las diferencias entre los sexos. Método: Se realizó un estudio observacional y correlacional con 225 adolescentes (108 chicos y 117 chicas), de entre los 12 y 17 años. Se realizaron medidas antropométricas, de composición corporal y la ratio 2D:4D. Resultados: se encontró una relación directa entre la ratio 2D:4D porcentaje de masa grasa $(r=, 321$ y $p<, 001)$ y con el IMC $(r=, 241$ y $\mathrm{p}<, 001)$. Al segmentar por sexo esta correlación solo se encontró en chicas $(r=, 408, p<, 001$ y $r=, 419, p<, 001$, respectivamente). Además, se encontraros diferencias significativas entre sexos para altura $(p=, 002)$, porcentaje de grasa $(p<, 001)$ y masa muscular $(p<, 001)$, ratio 2D:4D $(p<, 001)$. Conclusiones: existe una relación directa de la ratio 2D:4D con el porcentaje de masa grasa y el IMC en adolescentes de Extremadura; es decir, mayores ratios 2D:4D se asocian a mayores porcentajes de grasa e IMC. Además, las chicas presentan mayores ratios 2D:4D y porcentaje de masa grasa, y menor altura y masa muscular en comparación con los chicos.
\end{abstract}

\section{Abstract}

Introduction: The ratio 2D:4D is the quotient between the length of the index and ring finger of the hand, associated to the balance between androgens and estrogens, during the embryonic development. A low 2D:4D ratio reflects higher levels of testosterone in the uterus. This measure has been associated to certain body composition parameters, which could be indicative of the likelihood of health risks. Objectives: to analyze if there is a relationship between the 2D:4D ratio and body composition and to explore the differences between the sexes. Methods: An observational and correlational study was carried out with 225 adolescents (108 boys and 117 girls), between the ages of 12 and 17. Anthropometric, body composition and 2D:4D ratio measurements were made. Results: A direct relationship was found between the ratio 2D:4D fat mass percentage $(r=.321$ and $p<.001)$ and with the BMI $(r=.241$ and $p<.001)$. When segmented by sex, this correlation was only found in girls $(r=.408, p<.001$ and $r=.419, p<.001$, respectively). In addition, significant differences were found between sexes for height $(p=.002)$, fat mass percentage $(p<.001)$ and muscle mass $(p<.001)$, ratio $2 D: 4 D(p<.001)$. Conclusions: there is a direct relationship between the ratio 2D:4D and the fat mass percentage and BMI in adolescents from Extremadura; that is, higher ratios 2D:4D are associated with higher fat mass percentages and BMI. In addition, girls have higher 2D:4D ratios and fat mass percentage, and lower height and muscle mass compared to boys. 


\section{Introducción}

En la actualidad existen diversas medidas antropométricas que han servido para determinar diferentes características relacionadas con aspectos sociales, psicosociales, evolutivos o de salud. Algunas de estas medidas son: el dimorfismo facial, tomando algunos puntos faciales como referencia (Carré \& McCormick, 2008; Muñoz-Reyes, Gil-Burmann, \& Turiegano, 2014), la dermatoglifia, que consiste en el estudio de indicadores dactilares conseguidos mediante impresiones digitales de las manos (Avella \& Medellín, 2013; Juárez-Toledo, DomínguezGarcía, Laguna-Camacho, Sotomayor-Serrano, \& Balbás-Lara, 2018; Rodriguez, Montenegro, \& Petro, 2019) o la ratio 2D:4D, que viene determinada por el cociente entre las longitudes de los dedos índice (2D) y anular (4D). No obstante, es importante indicar que no es aconsejable utilizar estos métodos de forma aislada y sí en relación con otros.

Más específicamente, la relación 2D:4D viene dada por el balance entre los andrógenos y los estrógenos durante un pequeño espacio temporal del desarrollo embrionario (Zheng \& Cohn, 2011). Estudios previos informaron que los andrógenos del útero de la madre podrían estimular el crecimiento 4D, mientras que los estrógenos podrían estar relacionados con el crecimiento del 2D (Manning et al., 2000; Swaddle, 2002). Así, niveles altos de testosterona en el útero, en comparación con los niveles de estrógenos, se relacionan con una ratio 2D:4D baja; por lo que los hombres suelen presentar, ratios 2D:4D más bajos que las mujeres (Fink, Thanzami, Seydel, \& Manning, 2006; Hönekopp \& Schuster, 2010; Kociuba, Kozieł, Chakraborty, \& Ignasiak, 2017; Manning et al., 2000). Este dimorfismo sexual, comienza a partir de la novena semana de vida fetal (Malas, Dogan, Evcil, \& Desdicioglu, 2006), y se ve poco afectado por la pubertad, es decir, que la ratio 2D:4D no se ve influenciado por el crecimiento del individuo, siendo relativamente estable a lo largo de la vida (Mclntyre, Ellison, Lieberman, Demerath, \& Towne, 2005; Trivers, Manning, \& Jacobson, 2006). Por tanto, la ratio 2D:4D no difiere entre las diferentes etapas de desarrollo del individuo.

La ratio 2D:4D se constituye como una medida rápida, fácil, permanente, no invasiva, y de bajo coste de la exposición androgénica prenatal (Bermúdez, 2018). Estudios previos han mostrado que esta ratio, puede estar relacionada con ciertos parámetros de la composición corporal, que podrían ser indicativos de la probabilidad de presentar riesgos para la salud que pudieran estar asociado a una mala composición corporal (sobrepeso u obesidad, enfermedad coronaria, hipertensión arterial o diabetes mellitus) (Carvalho et al., 2015; González, Díaz, Mendizabal-Ruiz, Medina, \& Morales, 2014; Peltzer et al., 2014) y con ellos actuar de manera preventiva ante ellos. Por tanto, la ratio 2D:4D podría ser utilizada como herramienta complementaria para conocer la probabilidad de riesgos para la salud 
relacionados con una mala composición corporal, aportando así, mayor cantidad de datos y reforzando de esta manera las decisiones a tomar en relación con la detección diferentes patologías.

La ratio 2D:4D ha sido también estudiada en relación con diferentes aspectos como: el nivel de agresividad, trastornos psicosociales, y diferentes aspectos de la salud o la educación (Coates, Gurnell, \& Rustichini, 2009; Coco et al., 2011; Grimbos, Dawood, Burriss, Zucker, \& Puts, 2010; Romero-Martínez et al., 2013). En este sentido, Bailey y Hurd asociaron una ratio más baja con rangos más altos de puntuaciones en agresividad física en los hombres (Bailey \& Hurd, 2005); sin embargo, no encontraron relación en mujeres. Esto podría explicarse por la presencia de mayores niveles de testosterona en hombres, ya que, niveles más elevados de testosterona han sido asociados con mayores niveles de agresividad (Simpson, 2001). En este contexto, en relación con la ratio 2D:4D, como ya se ha indicado previamente que, menores ratios 2D:4D se asocian con niveles de testosterona más elevados (van der Meij, Almela, Buunk, Dubbs, \& Salvador, 2012).

La ratio 2D:4D ha sido también relacionada con diferentes trastornos imperantes en niños y adolescentes. Por ejemplo, Romero-Martínez et al. (Romero-Martínez et al., 2013), relacionaron la ratio 2D:4D con el Trastorno del Espectro Autista (TEA), mostrando que la ratio 2D:4D de los progenitores de personas con TEA, podría explicar los cocientes de empatía y autismo, conductas cooperativas y niveles de cortisol. En esta misma línea, Font-Jordá et al. (Font-Jordà, Gamundí, Llobera, \& Aguilar-Mediavilla, 2018) mostraron la relación inversa entre la ratio 2D:4D de la mano y el Trastorno Específico del Lenguaje en niños de entre 5 y 8 años. Así mismo, estudios previos mostraron la relación directa existente entre la ratio 2D:4D y los niveles séricos de estradiol en adolescentes de entre 8 y 15 años (Zhang et al., 2019) indicando que, una mayor ratio 2D:4D se asoció con mayores niveles séricos de estradiol y la relación inversa entre la ratio 2D:4D y la severidad prequirúrgica del cáncer de próstata, mostrando que menores ratios, se relacionaron con una mayor severidad (Sánchez-Rodríguez et al., 2018).

Por tanto, las observaciones expuestas anteriormente sugieren que las longitudes de los dígitos pueden servir como medida práctica indirecta del comportamiento y del estado de salud o enfermedad (Mayhew, Gillam, McDonald, \& Ebling, 2007). 
Dentro del ámbito de la salud, más específicamente, la ratio 2D:4D ha sido relacionada con la composición corporal. Estudios previos, mostraron que las personas con mayores ratios 2D:4D, presentaban un mayor porcentaje de grasa corporal (Agha-Alinejad, Farzad, Akbari, Moffit, \& Dakhili, 2019; Eler, 2018). También se ha analizado la relación entre la ratio 2D:4D y diferentes parámetros antropométricos como la altura, el peso, el índice de masa corporal (IMC), la circunferencia de la cintura (Eler, 2018; Erkec, 2019; Ranson, Stratton, \& Taylor, 2015) y la relación cintura-pecho (Fink, Neave, \& Manning, 2003). En líneas generales, la mayoría de autores informan de la existencia de una relación directa entre la ratio y el IMC, pero inversa entre la ratio y la altura. No obstante, otros estudios no encontraron ningún tipo de relación entre la ratio 2D:4D y algunas medidas antropométricas, como por ejemplo, el índice de masa corporal y la cintura en adultos (Muller et al., 2013). Estas contradicciones podrían derivarse del número de participantes sobre los que se realizaron los diferentes estudios.

En base a lo expuesto anteriormente, la ratio 2D:4D podría constituirse como medida útil, de obtención fácil, rápida y de bajo coste, estable y no invasiva, que podría servir como complemento a otras medidas, dando una visión inicial orientativa acerca de las relaciones mencionadas con anterioridad. En este sentido, podría ser interesante conocer si existe alguna relación entre la ratio 2D:4D y diferentes parámetros relacionados con la composición corporal en adolescentes; así como analizar si existen diferencias entre sexos, tanto en la ratio, como en la composición corporal. Teniendo en cuenta todo lo anterior, se plantearon las siguientes hipótesis: 1) menores ratios 2D:4D se relacionarán con menores niveles masa grasa e IMC y mayores niveles de masa muscular y 2) los chicos presentarán menores ratios 2D:4D, menor porcentaje de masa grasa y mayor cantidad de masa muscular en comparación con las chicas.

Por tanto, el presente estudio tuvo los siguientes objetivos: 1) explorar si existe alguna relación entre la ratio 2D:4D con el IMC, la masa muscular y el porcentaje de masa grasa en adolescentes de Extremadura y 2) analizar si existen diferencias entre sexos para estos parámetros.

\section{Método}

\section{Diseño del estudio}

Se realizó un estudio observacional y correlacional para obtener información sobre la composición corporal y la ratio 2D:4D en adolescentes de secundaria de Extremadura, encuadrado dentro del Observatorio de Bienestar, Obesidad y Motricidad Infantil y Juvenil en 
Extremadura (WOMO) (Mendoza-Muñoz et al., 2020b). La información recopilada se procesó para analizar las posibles relaciones y asociaciones entre las diferentes variables evaluadas y explorar si existen diferencias entre sexos para los diferentes parámetros evaluados.

\section{Aprobación ética}

La aprobación ética fue proporcionada por el Comité de Bioética y Bioseguridad de la Universidad de Extremadura el 18 de diciembre de 2019 (número de aprobación: 257/2019).

\section{Calculo muestral}

Se requiere un tamaño muestral de 70 para alcanzar una potencia estadística del 90\% para detectar una diferencia de -0,310 entre la correlación de hipótesis nula de 0,290 y la correlación de hipótesis alternativa de 0,60 utilizando una prueba de hipótesis de dos caras con un nivel de significación igual a 0,05.

\section{Participantes}

Para reclutar la muestra del presente estudio, se contactó con dos centros de Educación Secundaria de Extremadura, uno de la provincia de Cáceres y otro de la provincia de Badajoz. Se reclutaron un total de 225 adolescentes (108 chicos y 117 chicas), con edades comprendidas entre los 12 y los 17 años.

Los participantes debían cumplir los siguientes criterios de inclusión: 1) edad comprendida entre 12 y 17 años, 2) estar empadronados y/o residir en la comunidad autónoma de Extremadura, 3) estar autorizados por sus padres o tutores legales y 4) aceptar su participación voluntaria en el estudio.

Procedimiento, material y medida.

En este proyecto se utilizaron diversas herramientas para evaluar tanto la la ratio 2D:4D como la composición corporal de los participantes.

Ratio 2D:4D. La ratio 2D:4D se tomó utilizando un calibrador Vernier digital (Mitutoyo Co, Kanagawa, Japón) con una precisión de 0,01 mm. Se tomó como referencia la superficie ventral de la mano, y dentro de ella, la línea media del pliegue basal del dedo elegido, extendiéndose los dos extremos del calibrador por ella hasta llegar a la punta del mismo y sin ejercer presión en ella; tal y como describieron Manning, Scutt, Wilson, and Lewis-Jones (1998). 
Figura 1. Medición 2D:4D

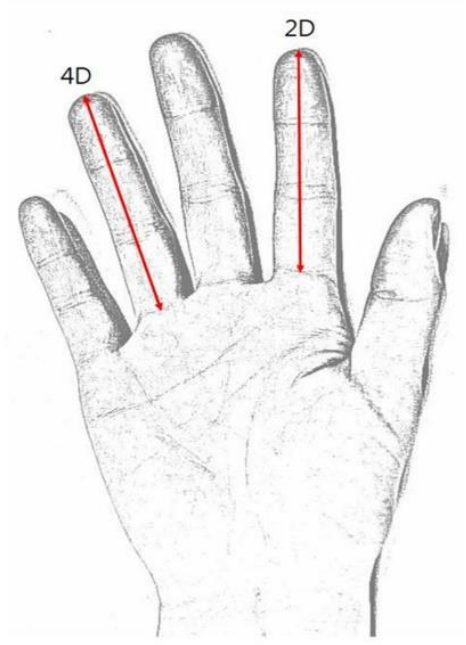

Nota. Tomado de Why is digit ratio correlated to sports performance? (p.515), por Kim \& Kim, 2016, Journal of exercise rehabilitation.

Medidas antropométricas y composición corporal. Se realizaron en condiciones estandarizadas, siguiendo en todo momento el protocolo establecido en el Manual de Procedimiento de Recopilación de Datos, elaborado específicamente para la iniciativa Childhood Obesity Surveillance Initiative (COSI) (Wijnhoven et al., 2014). Antes de tomar cualquiera de las medidas, se pidió a los participantes que se quitaran los zapatos y calcetines, así como cualquier ropa pesada (abrigos, suéteres, chaquetas, etc.) que portaran. Además, se les pidió que vaciaran sus bolsillos y se quitaran los cinturones y otros accesorios o complementos que pudiesen llevar. La altura se midió con un tallímetro (Tanita Tantois, Tanita Corporation, Tokyo, Japón). El tallímetro se colocó en una superficie vertical de manera que la escala de medida estaba perpendicular al suelo. La talla se medió en bipedestación, con los hombros equilibrados y los brazos relajados a lo largo del cuerpo. La medida se tomó en cm, hasta el mm más cercano. El peso se midió con un bioimpedanciómetro (Tanita MC-780 MA, Tanita Corporation, Tokyo, Japón) y se registró en kilogramos, hasta los 100 g más próximos. Este mismo instrumento se utilizó para evaluar la masa grasa y la masa muscular. La evaluación se realizó en el "modo estándar", introduciendo la edad, el sexo y la altura de cada participante. Se siguieron todas las recomendaciones para asegurar la validez y fiabilidad de los datos tomados por el bioimpedanciómetro (Sgroi \& De Lorenzo, 2011; TANITA, 2015): realizar la prueba más de 3 horas después de levantarse, orinar antes de la medida, no comer ni beber durante las 3 horas previas a la prueba, no comer ni beber en exceso el día anterior, no hacer ejercicio pesado ni beber alcohol durante las últimas 12 horas y no portar objetos metálicos o poseer marcapasos. Este instrumento ha sido previamente validado en la 
población objetivo comparando sus resultados con la densitometría (CCl: 0,84) (Verney, Schwartz, Amiche, Pereira, \& Thivel, 2015).

\section{Análisis estadístico}

Toda la información recopilada se tabuló en una base de datos anonimizada y diseñada específicamente para este estudio. Los análisis estadísticos se llevaron a cabo utilizando el software IBM SPSS Statistics (Version 21, IBM SPSS, Chicago, IL, USA).

Los datos se presentan como media y desviación típica; así como, mediana y rango intercuartílico, tanto para la muestra total como para las submuestras divididas por género (chicos y chicas). En primer lugar, se comprobó la normalidad y homogeneidad de los datos usando las pruebas de Kolmogorov-Smirnov y Levene, respectivamente. Posteriormente, las diferencias entre sexos se analizaron utilizando la prueba $T$ de Student para muestras independientes en el caso de las variables paramétricas (variables de distribución normal): altura y porcentaje de grasa; y la prueba U Mann-Whitney para las variables no paramétricas (variables de distribución no normal): edad, peso, IMC, masa muscular y ratio 2D:4D. Se consideraron diferencias significativas para $\mathrm{p} \leq 0,05$.

Adicionalmente, para cuantificar las relaciones entre las diferentes variables, se aplicaron los coeficientes de correlación de Pearson (variables paramétricas) y Spearman (variables no paramétricas). Se aplicó la corrección de Bonferroni a partir la fórmula $\alpha^{*}=\alpha / n-1$ (McLaughlin \& Sainani, 2014), donde $\alpha^{\star}$ es el valor corregido con el cual se debe rechazar la hipótesis nula y n es la cantidad de pares de hipótesis. Por tanto, el nivel de significación alfa se fijó en 0,025 para las comparaciones múltiples de la ratio 2D:4D con la composición corporal. Los valores de correlación se interpretaron siguiendo los umbrales de clasificación de Cohen (Cohen, 1988): < 0,2 muy baja o casi nula; 0,2 a < 0,4 baja; 0,4 < 0,6 moderada; 0,6 a < 0,8 alta; $\geq 0,8$ muy alta o casi perfecta.

\section{Resultados}

El número total de participantes fue de 225 , siendo similar el número de chicos y chicas participantes en el estudio (48\% chicos versus $52 \%$ chicas).

La Tabla 1 muestra la caracterización de los participantes y la comparación entre ambos sexos para la edad, las variables antropométricas y de composición corporal y la ratio 2D:4D. Los resultados mostraron diferencias estadísticamente significativas a favor de los chicos en la altura $(p=, 002)$, el porcentaje de masa grasa $(p<, 001)$ y masa muscular $(p<, 001)$. Además, se 
encontraron diferencias estadísticamente significativas en la ratio 2D:4D de la mano derecha, siendo esta menor en los chicos ( $p<, 001)$.

Tabla 1. Caracterización y diferencias significativas entre sexos en las variables de composición corporal y dimensiones de los dedos.

\begin{tabular}{|c|c|c|c|c|}
\hline & & Chicos & Chicas & $p$ \\
\hline $\mathrm{N}(\%)$ & & $108(48)$ & $117(52)$ & \\
\hline \multirow{2}{*}{ Edad (años) } & Media (DT) & $13,59(1,32)$ & $13,72(1,51)$ & \multirow{2}{*}{, 758} \\
\hline & Mediana (RI) & $13,00(1,00)$ & $13,00(1,00)$ & \\
\hline \multirow{2}{*}{ Peso (kg) } & Media (DT) & $54,14(14,06)$ & $51,44(9,37)$ & \multirow{2}{*}{, 130} \\
\hline & Mediana (RI) & $52,9(20,9)$ & $49,80(11,10)$ & \\
\hline \multirow{2}{*}{ Altura (cm) } & Media (DT) & $161,80(10,71)$ & $158,03(7,03)$ & \multirow{2}{*}{, 002} \\
\hline & Mediana (RI) & $163,50(15,75)$ & $158,00(8,00)$ & \\
\hline \multirow{2}{*}{ IMC $\left(\mathrm{m} \cdot \mathrm{kg}^{-2}\right)$} & Media (DT) & $20,32(3,68)$ & $20,52(3,04)$ & \multirow{2}{*}{, 367} \\
\hline & Mediana (RI) & $19,80(5,40)$ & $20,40(3,85)$ & \\
\hline \multirow{2}{*}{$\begin{array}{l}\text { Porcentaje de Grasa } \\
\text { (\%) }\end{array}$} & Media (DT) & $19,99(6,00)$ & $27,00(5,08)$ & \multirow{2}{*}{$<, 001$} \\
\hline & Mediana (RI) & $18,35(9,55)$ & $26,10(6,55)$ & \\
\hline \multirow{2}{*}{ Masa Muscular (kg) } & Media (DT) & $40,67(9,34)$ & $35,31(4,97)$ & \multirow{2}{*}{$<, 001$} \\
\hline & Mediana (RI) & $40,15(15,40)$ & $34,70(6,75)$ & \\
\hline \multirow{3}{*}{ 2D:4D (cm) } & Media (DT) & $0,94(0,05)$ & $0,96(0,42)$ & \multirow{3}{*}{$<, 001$} \\
\hline & Mediana (RI) & $0,92(0,06)$ & $0,95(0,06)$ & \\
\hline & Mediana (RI) & $7,05(0,44)$ & $6,79(0,54)$ & \\
\hline
\end{tabular}

2D:4D: ratio 2D:4D de la mano derecha; 2D: medida del dedo índice; 4D: medida del dedo anular. N: tamaño de muestral. Los datos se muestran como media (Desviación Típica) y Mediana (Rango Intercuartílico). Se consideraron diferencias significativas cuando $p \leq 0,05$.

La Tabla 2 muestra la relación existente entre la ratio 2D:4D de la mano derecha con las variables de composición corporal. En general, los resultados mostraron una correlación directa de la ratio 2D:4D con el IMC y el porcentaje de masa grasa, lo que significa que una menor ratio 2D:4D se asocia con menores valores de IMC y porcentaje de grasa.

Resultados similares se encontraron tanto para el IMC como para el porcentaje de masa grasa en las chicas, al segmentar los datos por sexo. En cambio, no se encontraron correlaciones significativas entre ninguna variable antropométrica ni de composición corporal con la ratio 2D:4D. 
Tabla 2. Relación entre la ratio 2D:4D y la composición corporal.

\begin{tabular}{|c|c|c|c|}
\hline & IMC & Masa Muscular & Masa Grasa (\%) \\
\hline \multicolumn{4}{|l|}{ Todos } \\
\hline $2 \mathrm{D}: 4 \mathrm{D}$ &, $241^{\star \star}$ &,- 011 & $321^{\star \star}$ \\
\hline \multicolumn{4}{|l|}{ Chicos } \\
\hline 2D:4D &, 186 &, 133 &, 145 \\
\hline \multicolumn{4}{|l|}{ Chicas } \\
\hline 2D:4D & $419^{* *}$ &, 036 &, $408^{\star \star}$ \\
\hline $\begin{array}{l}\text { 2D:4D: ratio 2D: } \\
{ }^{\star} \text { Correlación signi }\end{array}$ & $\begin{array}{l}\text { nano derecha. } \\
\text { a a nivel,01 }\end{array}$ & *Correlación sign & tiva a nivel ,025; \\
\hline
\end{tabular}

\section{Discusión}

Los principales hallazgos del presente estudio muestran que los chicos presentan una mayor altura y masa muscular; mientras que las chicas muestran un porcentaje de grasa y una ratio 2D:4D mayor, al comparar ambos sexos. Además, se encontró una relación directa entre la ratio 2D:4D con el IMC y el porcentaje de masa grasa al considerar ambos sexos. No obstante, al segmentar por sexo, solo se mantuvo dicha relación en las chicas. En cambio, no se encontrón diferencias entre sexos ni para el peso, ni para el IMC; así como, tampoco se obtuvo relación entre la ratio 2D:4D y la masa muscular.

Los resultados de este estudio concuerdan con los reportados por estudios previos que mostraron diferencias significativas entre sexos en diferentes parámetros de composición corporal en población adolescente (Castro-Piñero et al., 2009; Kirchengast, 2010; MendozaMuñoz et al., 2020a; Ortega et al., 2011) y que no encontraron diferencias en el IMC (MendozaMuñoz et al., 2020a; Ortega et al., 2011). De igual forma, en relación a las diferencias significativas encontradas en la ratio 2D:4D entre chicos y chicas $(p<.001)$, diversos estudios abalan esta teoría en numerosas poblaciones (Butovskaya, Fedenok, Burkova, \& Manning, 2013; Fink, Manning, \& Neave, 2006; Hampson, Ellis, \& Tenk, 2008; Hönekopp \& Schuster, 2010; Kociuba et al., 2017; Manning et al., 2000; Putz, Gaulin, Sporter, \& McBurney, 2004; Ranson et al., 2015), como por ejemplo, en escolares (Ranson et al., 2015), adolescentes (Butovskaya et al., 2013), o adultos (Hampson et al., 2008; Putz et al., 2004). Esto puede ser explicado por la corriente que afirma que la ratio 2D:4D no se ve afectada por el crecimiento (McIntyre et al., 2005; Trivers et al., 2006). Concretamente, autores como Manning et al. (2000) 
o Swaddle (2002) indicaron que el crecimiento prenatal del cuarto dedo estaría estimulado por los andrógenos; mientras que, el segundo dedo lo estaría por los estrógenos. Esto podría explicar que los chicos presenten una ratio significativamente menor que chicas y, ya que, una baja proporción en la ratio 2D:4D, refleja niveles más altos de testosterona en el útero, en comparación con los niveles de estrógenos (Malas et al., 2006).

La ratio $2 \mathrm{D}: 4 \mathrm{D}$ ha sido mostrada como un biomarcador relacionado con las medidas antropométricas (Erkec, 2019; Fink et al., 2003). En este sentido, los resultados del presente estudio muestran que existe una asociación entre la ratio 2D:4D tanto con el porcentaje de masa grasa $(r=, 321, p<, 001)$, como con el IMC $(r=, 241, p<, 001)$. No obstante, dicha relación difiere ligeramente al segmentar por sexos, ya que, la relación de la ratio 2D:4D con el porcentaje de masa grasa e IMC se mantiene las chicas $(r=, 408, p<, 001$ y $r=, 419, p<, 001$, respectivamente), pero no existe en los chicos $(r=, 145, p=, 135$ y $r=, 186, p=, 054$ respectivamente). Estos resultados coinciden con los reportados en estudios realizados previamente como los de Ranson et al. (2015), Agha-Alinejad et al. (2019) y Eler (2018). Concretamente, Agha-Alinejad et al. (2019) concluyeron que existía una relación directa entre la ratio $2 \mathrm{D}: 4 \mathrm{D}$ y el porcentaje de masa grasa $(r=, 132 ; p<, 05)$ en niñas con una edad ligeramente inferior (8-12 años). Respecto a la relación entre la ratio y el IMC, estudios previos mostraron también una relación directa (Eler, 2018; Ranson et al., 2015), sumado al de otras variables como la estatura y la circunferencia de cintura, no existiendo relación en el caso de los niños (Ranson et al., 2015), en línea con los resultados del presente estudio. Esta relación directa entre la ratio 2D:4D y la masa grasa puede ser debida a que los estrógenos estimulan la multiplicación de las células grasas (Mauras et al., 2015), lo que apoyaría los resultados obtenidos, donde un mayor ratio 2D:4D se relaciona con una mayor porcentaje de grasa.

Adicionalmente, estudios previos observaron una relación negativa entre la ratio 2D:4D y la masa muscular (Halil et al., 2013; Kociuba et al., 2018; Pruszkowska-Przybylska et al., 2018), que no fue obtenida en el presente estudio; a pesar de que, la testosterona ha sido relacionada positivamente con la producción de tejido muscular (Lin et al., 2012), y por tanto, una menor ratio implicaría una mayor cantidad de tejido muscular.

En este sentido, las relaciones establecidas entre la ratio y los parámetros de composición corporal, sugieren que la ratio 2D:4D podría ser utilizada como herramienta complementaria para la obtención de información inicial sobre la predisposición a presentar una mayor o menor porcentaje de masa grasa y/o IMC, desde el nacimiento; ya que como se mencionaba 
anteriormente dicha ratio es estable a lo largo de la vida (Mclntyre et al., 2005; Trivers et al., 2006). Esto permitiría actuar de manera preventiva ante los diferentes riesgos para la salud o patologías asociados a la presencia de mayor cantidad de grasa o un alto IMC, como pueden ser sobrepeso u obesidad, enfermedad coronaria, hipertensión arterial o diabetes mellitus (Carvalho et al., 2015; González et al., 2014; Peltzer et al., 2014). No obstante, los resultados obtenidos deben tomarse con cautela, ya que la ratio 2D:4D podría aportar una visión inicial en relación con dicha predisposición, la cual debería ser confirmada con herramienta específicas para la valoración de la composición corporal.

Es necesario indicar que el presente estudio cuenta con una serie de limitaciones. Aunque el calibrador Vernier es un instrumento fiable (CCI mano derecha:0,77), Allaway, Bloski, Pierson, and Lujan (2009) propone otras técnicas con las que se podría mejorar la calidad del estudio, por ejemplo, mediante la utilización del escáner Hewlett Packard ScanJet 3C (CCl mano derecha:0,79), el uso de la fotocopiadora Xerox Copycenter C35 (CCI mano derecha:0,87) o el Programa de manipulación y edición de imágenes GNU; GIMP versión 2.217 (CCl mano derecha:0,91). Por tanto, podría ser interesante realizar estudios futuros para analizar las diferencias entre las diferentes técnicas de medición propuestas. Otra de las limitaciones, podría ser el uso del bioimpedanciómetro para la evaluación de la composición corporal, en lugar de densitometría ósea, considerado el "gold estándar". No obstante, el bioimpedanciómetro utilizado (Tanita MC-780 MA, Tanita Corporation, Tokyo, Japón) ha demostrado un gran acuerdo y concordancia en la población objetivo (CCl > 0,99) (Verney et al., 2016). Finalmente, cabe mencionar que basamos nuestros hallazgos en una muestra representativa de los participantes en Extremadura, pero un tamaño muestral mayor podría mejorar la fuerza de las asociaciones y clarificar los resultados.

\section{Conclusión}

Con base a los resultados del presente estudio se puede concluir que existe una relación directa de la ratio 2D:4D con el porcentaje de masa grasa y el IMC en adolescentes de Extremadura; concretamente, que mayores ratios 2D:4D se asocian a mayores porcentajes de grasa e IMC. Sin embargo, al segmentar por sexo, dicha correlación solo se cumple en las chicas. Además, podemos afirmar que las chicas presentan mayores ratios 2D:4D y porcentaje de masa grasa, y menor altura y masa muscular en comparación con los chicos. 


\section{Agradecimientos}

Los autores desean agradecer a las escuelas y profesionales que permitieron el acceso para llevar a cabo este estudio.

\section{Conflicto de interés}

Los autores declaran no tener conflicto de interés.

\section{Financiación}

Esta investigación está financiada por el Servicio Público de Empleo de Extremadura (SEXPE), número de subvención TE-0009-18. Los financiadores no desempeñaron ningún papel en el diseño del estudio, la decisión de publicar o la preparación del manuscrito.

\section{Referencias}

Agha-Alinejad, H., Farzad, B., Akbari, M., Moffit, D. M., \& Dakhili, A. (2019). Digit ratios and motor and health-related fitness in pre-adolescent females. Annals of human biology, 46(3), 225-230.

Allaway, H. C., Bloski, T. G., Pierson, R. A., \& Lujan, M. E. (2009). Digit ratios (2D: 4D) determined by computer-assisted analysis are more reliable than those using physical measurements, photocopies, and printed scans. American Journal of Human Biology: The Official Journal of the Human Biology Association, 21(3), 365-370.

Avella, R. E., \& Medellín, J. P. (2013). Perfil dermatoglífico y somatotípico de atletas de la selección colombia de atletismo (velocidad) participante en los juegos panamericanos de Guadalajara, 2011.

Bailey, A. A., \& Hurd, P. L. (2005). Finger length ratio (2D: 4D) correlates with physical aggression in men but not in women. Biological psychology, 68(3), 215-222.

Bermúdez, S. R. (2018). ÍNDICE DE LONGITUD DIGITAL 2D: 4D EN LA DETECCIÓN E IDENTIFICACIÓN DE TALENTOS PARA DIFERENTES GRUPOS DE DEPORTE. Expomotricidad.

Butovskaya, M., Fedenok, J., Burkova, V., \& Manning, J. (2013). Sex differences in 2D: 4D and aggression in children and adolescents from five regions of Russia. American journal of physical anthropology, 152(1), 130-139.

Carré, J. M., \& McCormick, C. M. (2008). In your face: facial metrics predict aggressive behaviour in the laboratory and in varsity and professional hockey players. Proceedings of the Royal Society B: Biological Sciences, 275(1651), 2651-2656. 
Carvalho, C. A. d., Fonseca, P. C. d. A., Barbosa, J. B., Machado, S. P., Santos, A. M. d., \& Silva, A. A. M. d. (2015). The association between cardiovascular risk factors and anthropometric obesity indicators in university students in São Luís in the State of Maranhão, Brazil. Ciência \& Saúde Coletiva, 20, 479-490.

Castro-Piñero, J., González-Montesinos, J. L., Mora, J., Keating, X. D., Girela-Rejón, M. J., Sjöström, M., \& Ruiz, J. R. (2009). Percentile values for muscular strength field tests in children aged 6 to 17 years: influence of weight status. The Journal of Strength \& Conditioning Research, 23(8), 2295-2310.

Coates, J. M., Gurnell, M., \& Rustichini, A. (2009). Second-to-fourth digit ratio predicts success among high-frequency financial traders. Proceedings of the National Academy of Sciences, 106(2), 623-628.

Coco, M., Perciavalle, V., Maci, T., Nicoletti, F., Di Corrado, D., \& Perciavalle, V. (2011). The second-to-fourth digit ratio correlates with the rate of academic performance in medical school students. Molecular medicine reports, 4(3), 471-476.

Cohen, J. (1988). Statistical power analysis for the behavioral sciences: Jacob Cohen. J Am Stat Assoc, 84(363), 19-74.

Eler, N. (2018). The correlation between right hand finger ratio (2D: 4D) and the parameters of anthropometric and physical fitness in children. Journal of Human Sciences, 15(1), 656-664.

Erkec, O. E. (2019). Relationships Between the 2D: 4D Digit Ratio, Waist Circumference, Hand Preferences, Weight, Height, Waist-to-Height Ratio and BMI in a Turkish Population. International Journal of Morphology, 37(4).

Fink, B., Manning, J., \& Neave, N. (2006). The 2nd-4th digit ratio (2D: 4D) and neck circumference: implications for risk factors in coronary heart disease. International Journal of Obesity, 30(4), 711-714.

Fink, B., Neave, N., \& Manning, J. (2003). Second to fourth digit ratio, body mass index, waistto-hip ratio, and waist-to-chest ratio: their relationships in heterosexual men and women. Annals of human biology, 3066), 728-738.

Fink, B., Thanzami, V., Seydel, H., \& Manning, J. T. (2006). Digit ratio and hand - grip strength in German and Mizos men: Cross - cultural evidence for an organizing effect of prenatal testosterone on strength. American Journal of Human Biology: The Official Journal of the Human Biology Association, 18(6), 776-782.

Font-Jordà, A., Gamundí, A., Llobera, M. C. N., \& Aguilar-Mediavilla, E. (2018). Uso del índice digital D2: D4 como indicador biológico del trastorno específico del lenguaje. Paper presented at the Anales de Pediatría. 
González, C. S., Díaz, Y. B., Mendizabal-Ruiz, A., Medina, E. D., \& Morales, J. (2014). Prevalence of obesity and altered lipid profile in university students. Nutricion hospitalaria, 29(2), 315-321.

Grimbos, T., Dawood, K., Burriss, R. P., Zucker, K. J., \& Puts, D. A. (2010). Sexual orientation and the second to fourth finger length ratio: a meta-analysis in men and women. Behavioral neuroscience, 124(2), 278.

Halil, M., Gurel, E. I., Kuyumcu, M. E., Karaismailoglu, S., Yesil, Y., Ozturk, Z. A., . . Arıogul, S. (2013). Digit (2D: 4D) ratio is associated with muscle mass (MM) and strength (MS) in older adults: Possible effect of in utero androgen exposure. Archives of gerontology and geriatrics, 56(2), 358-363.

Hampson, E., Ellis, C. L., \& Tenk, C. M. (2008). On the relation between 2D: 4D and sexdimorphic personality traits. Archives of sexual behavior, 37(1), 133.

Hönekopp, J., \& Schuster, M. (2010). A meta-analysis on 2D: 4D and athletic prowess: Substantial relationships but neither hand out-predicts the other. Personality and Individual Differences, 48(1), 4-10.

Juárez-Toledo, L., Domínguez-García, M., Laguna-Camacho, A., Sotomayor-Serrano, N., \& Balbás-Lara, F. (2018). Somatotype and digital dermatoglyphic in Mexican football players. Revista Internacional de Medicina y Ciencias de la Actividad Fisica y del Deporte, 18(70).

Kim, T. B., \& Kim, K. H. (2016). Why is digit ratio correlated to sports performance? Journal of exercise rehabilitation, 12(6), 515.

Kirchengast, S. (2010). Gender differences in body composition from childhood to old age: an evolutionary point of view. Journal of Life Sciences, 2(1), 1-10.

Kociuba, M., Ignasiak, Z., Sebastjan, A., Kochan, K., Cichy, I., Dudkowski, A., ... Kozieł, S. (2018). Sex differences in relationship between body composition and digit length ratio (2D: 4D) in students of military courses. AnthropologicAl review, 81(4), 393-403.

Kociuba, M., Kozieł, S., Chakraborty, R., \& Ignasiak, Z. (2017). Sports preference and digit ratio (2D: 4D) among female students in Wrocław, Poland. Journal of biosocial science, 49(5), 623-633.

Lin, S. L., Lee, S. L., Hui, L. L., Au Yeung, S. L., Tse, M. A., Leung, G. M., \& Schooling, C. M. (2012). Pubertal muscle mass and diabetes markers in Chinese adolescents. American Journal of Human Biology, 24(2), 183-185.

Malas, M. A., Dogan, S., Evcil, E. H., \& Desdicioglu, K. (2006). Fetal development of the hand, digits and digit ratio (2D: 4D). Early human development, 82(7), 469-475.

Manning, J. T., Barley, L., Walton, J., Lewis-Jones, D., Trivers, R., Singh, D., . . Henzi, P. (2000). The 2nd: 4th digit ratio, sexual dimorphism, population differences, and reproductive 
success: evidence for sexually antagonistic genes? Evolution and Human Behavior, 2/(3), 163-183.

Manning, J. T., Scutt, D., Wilson, J., \& Lewis-Jones, D. I. (1998). The ratio of 2nd to 4th digit length: a predictor of sperm numbers and concentrations of testosterone, luteinizing hormone and oestrogen. Human Reproduction (Oxford, England), 13(11), 3000-3004.

Mauras, N., Santen, R. J., Colón-Otero, G., Hossain, J., Wang, Q., Mesaros, C., \& Blair, I. A. (2015). Estrogens and their genotoxic metabolites are increased in obese prepubertal girls. The Journal of Clinical Endocrinology \& Metabolism, 1006), 2322-2328.

Mayhew, T., Gillam, L., McDonald, R., \& Ebling, F. (2007). Human 2D (index) and 4D (ring) digit lengths: their variation and relationships during the menstrual cycle. Journal of Anatomy, 211(5), 630-638.

Mclntyre, M. H., Ellison, P. T., Lieberman, D. E., Demerath, E., \& Towne, B. (2005). The development of sex differences in digital formula from infancy in the Fels Longitudinal Study. Proceedings of the Royal Society B: Biological Sciences, 272(1571), 1473-1479.

McLaughlin, M. J., \& Sainani, K. L. (2014). Bonferroni, Holm, and Hochberg corrections: fun names, serious changes to $p$ values. $P M \& R$ : the journal of injury, function, and rehabilitation, 6(6), 544.

Mendoza-Muñoz, M., Adsuar, J. C., Pérez-Gómez, J., Muñoz-Bermejo, L., Garcia-Gordillo, M. Á., \& Carlos-Vivas, J. (2020a). Influence of body composition on physical fitness in adolescents. Medicina, 56(7), 328.

Mendoza-Muñoz, M., Adsuar, J. C., Pérez-Gómez, J., Muñoz-Bermejo, L., Garcia-Gordillo, M. Á., \& Carlos-Vivas, J. (2020b). Well-Being, Obesity and Motricity Observatory in Childhood and Youth (WOMO): A Study Protocol. International journal of environmental research and public health, 17(6), 2129.

Muller, D. C., Manning, J. T., Hopper, J. L., English, D. R., Giles, G. G., \& Severi, G. (2013). No strong association between second to fourth digit ratio (2D: 4D) and adult anthropometric measures with emphasis on adiposity. Annals of human biology, 40(2), 201-204.

Muñoz-Reyes, J.-A., Gil-Burmann, C., \& Turiegano, E. (2014). Digit Ratio 2D: 4D, facial masculinization and aggressiveness in Spanish adolescents/Índice 2D: 4D, masculinización facial y agresividad en adolescentes españoles. Estudios de Psicología, 35(2), 319-340.

Ortega, F. B., Artero, E. G., Ruiz, J. R., España-Romero, V., Jiménez-Pavón, D., VicenteRodríguez, G., ... Ottevaere, C. (2011). Physical fitness levels among European adolescents: the HELENA study. British journal of sports medicine, 45(1), 20-29. 
Peltzer, K., Pengpid, S., Samuels, T., Özcan, N. K., Mantilla, C., Rahamefy, O. H., .. Gasparishvili, A. (2014). Prevalence of overweight/obesity and its associated factors among university students from 22 countries. International journal of environmental research and public health, 11(7), 7425-7441.

Pruszkowska-Przybylska, P., Sitek, A., Rosset, I., Sobalska-Kwapis, M., Słomka, M., Strapagiel, D., \& Żądzińska, E. (2018). Association of the 2D: 4D digit ratio with body composition among the Polish children aged 6-13 years. Early human development, 124, $26-32$.

Putz, D. A., Gaulin, S. J., Sporter, R. J., \& McBurney, D. H. (2004). Sex hormones and finger length: What does 2D: 4D indicate? Evolution and Human Behavior, 25(3), 182-199.

Ranson, R., Stratton, G., \& Taylor, S. (2015). Digit ratio (2D: 4D) and physical fitness (Eurofit test battery) in school children. Early human development, 91(5), 327-331.

Rodriguez, A. N., Montenegro, O., \& Petro, J. L. (2019). Perfil dermatoglífico y somatotipificación de jugadores adolescentes de fútbol (Dermatoglyphic profile and somatotyping of adolescent soccer players). Retos, 36(36), 32-36.

Romero-Martínez, Á., de Andrés-García, S., Sariñana-González, P., Sanchis-Calatayud, M., Roa, J. M., González-Bono, E., \& Moya-Albiol, L. (2013). The 2D: 4D ratio and its relationship with other androgenisation parameters in parents of individuals with autism spectrum disorders. anales de psicología, 29(1), 264-271.

Sánchez-Rodríguez, C., Oñate-Celdrán, J., García-Escudero, D., Samper-Mateo, P., VilchezCostas, A., Torres-Roca, M., ... Mendiola-Olivares, J. (2018). Relación entre ratio digital (2D: 4D) y severidad preoperatoria del cáncer de próstata. Revista Urología Colombiana/Colombian Urology Journal, 27(03), 254-259.

Sgroi, M., \& De Lorenzo, A. (2011). Stato Nutrizionale, Dieta Mediterranea e Attivita'Fisica: Analisi e Valutazione Della Composizione Corporea e Dello Stile di Vita di Una Popolazione Scolastica fra gli i 18 Anni. Roma: Casa Editrice Scolastica Lombardi. Edizioni scientifiche.

Simpson, K. (2001). The role of testosterone in aggression. McGill Journal of Medicine, 6(1), 3240.

Swaddle, J. P. (2002). Digit ratio: a pointer to fertility, behavior, and health. Heredity, 895), 403-403.

TANITA. (2015). Body composition analyzer MC-780MA Instruction Manual. (pp. 1-36).

Trivers, R., Manning, J., \& Jacobson, A. (2006). A longitudinal study of digit ratio (2D: 4D) and other finger ratios in Jamaican children. Hormones and Behavior, 492), 150-156.

van der Meij, L., Almela, M., Buunk, A. P., Dubbs, S., \& Salvador, A. (2012). 2 D: 4 D in Men Is Related to Aggressive Dominance but Not to Sociable Dominance. Aggressive behavior, 38(3), 208-212. 
Verney, J., Schwartz, C., Amiche, S., Pereira, B., \& Thivel, D. (2015). Comparisons of a multifrequency bioelectrical impedance analysis to the dual-energy $X$-ray absorptiometry scan in healthy young adults depending on their physical activity level. Journal of human kinetics, 47(1), 73-80.

Wijnhoven, T. M., van Raaij, J. M., Spinelli, A., Starc, G., Hassapidou, M., Spiroski, I., . . . Hovengen, R. (2014). WHO European Childhood Obesity Surveillance Initiative: body mass index and level of overweight among 6-9-year-old children from school year 2007/2008 to school year 2009/2010. BMC public health, 14(1), 806.

Zhang, Y., Zhou, Y., Li, T., Li, Z., Wu, L., Meng, Y., . . Fu, L. (2019). The association between digit ratio (2D: 4D) and blood pressure among children and adolescents. Hypertension Research, 42(6), 876-882.

Zheng, Z., \& Cohn, M. J. (2011). Developmental basis of sexually dimorphic digit ratios. Proceedings of the National Academy of Sciences, 108(39), 16289-16294. 